\title{
Secondary Information Fault-tolerance Method for Smart Substation Based on Residuals of Measurement Equations
}

\author{
Yutao Qiu ${ }^{1}$, Yimin Ding ${ }^{2}$, Wulue Pan ${ }^{1}$ and Chaoli Feng ${ }^{3}$ \\ ${ }^{1}$ State Grid Zhejiang Electric Power Company. Hangzhou, China \\ ${ }^{2}$ State Grid Jiaxing Power Supply Company. Jiaxing, China \\ ${ }^{3}$ State Grid Haiyan Power Supply Company. Jiaxing, China
}

\begin{abstract}
This paper presented a method of fault-tolerance for secondary-system information in smart substations. It was to solve the problem of information redundancy. This article drew on a method of state estimation on the primary-system and used the calculation of measurement-equation residuals to identify and eliminate bad data to complete the information fault-tolerance function. The diagrams illustrated the process of information interaction in the secondary-system, and introduced the configuration mode of relay protection in smart substations. Through simulation and verification, using the method of residual identification to perform protection fault-tolerance can effectively prevent the protection against misoperation caused by bad data, improve the credibility of data, and promote the reliability of the protection.
\end{abstract}

Keywords—smart substation; state estimation; residual identification; bad data distinguishing; information fault-tolerance

\section{INTRODUCTION}

Relay protection is the first line of defense in the power system and is responsible for the heavy responsibility for quickly removing faulty components. Its reliability is a fundamental requirement and has long been a focus on attention. In terms of improving the reliability of the protection device itself, various methods such as redundant configuration of software and hardware, design of anti-electromagnetic interference, and device self-checking are adopted. In engineering applications, reliability can be further improved on the protection for dual configurations. These methods are achieved with good effects[1-2], but when the CT sampling error and other sampling value data distortion occurs, it will directly lead to the problem of malfunction of the protection that has not been well resolved.

With the extensive implementation of smart substation technology, sampling methods and information in substations have gradually been digitized and networked. The typical smart substation protection system structure includes "Directsampling\& Direct-tripping" and "Direct-sampling\& Tripping over the network" according to the protection sampling method and action method, with common features that achieving digital sampling, sharing information and implementing applications of information on the network, in which centralizing information to provide hardware supports for the secondary-function optimization of the substation. However, the reliability analysis shows that the electronic transformer adopts many new electronic components. Regardless of the structure of the protection system, the increase in the number of intermediate devices will cause the reliability of the protection device to decline, and the distortion of the sampling data is more likely to occur along with security risks[3].

Current power system state estimation analysis is based on the primary system data and the redundancy relationship between them. By collecting analog quantity, such as phase voltage, phase current plus phase power, and network topologies, combined with primary system power flow calculation, equivalent calculation, other physical constraints and modeling method, we can form a primary system state estimation and secondary system protection information fault-tolerance system[4-6].In order to adapt to the substation intelligence, interconnected power grids getting large and access to new energy sources, many literatures have carried out secondary system information fault-tolerance studies, including the method of state estimation for information redundancy and structural redundancy in secondary-systems of smart substations, which improving the accuracy and speed of the state estimation. In order to adapt to the substation intelligence, large-scale interconnected power grids and access to new energy sources, many literatures have carried out state estimation studies, including the method of state estimation for information redundancy and structural redundancy in secondary systems of smart substations. The accuracy and speed of the state estimation are improved. However, due to the sampling and calculation speed of the state estimation and failures changing the network topology, the state estimation and residual analysis method has not been applied to the protection sampling information.

The state estimation method essentially relies on redundant information to utilize the basic constraint equations of the power system to improve the information accuracy, and it has the ability to identify error information. The basic relation of the redundant information in the substation is shown in Figure I, but the information in the devices such as the measurement and control device, energy metering and fault recorder is not realtime. Although the state estimation methods based on this information have high information redundancy, they can't meet the requirements of the protection towards information[7]. This article draws on the idea of state estimation, utilizes the advantages of digitization and network sharing of intelligent substation information sampling, identifies error sampling information in the station through residual calculations of the 
measurement equation, and proposes a new method for preventing misoperation of relay protection devices in smart substations.

$\begin{array}{ccc}\text { Measurement } & \\ \text { and control } & \text { Redundant } \\ \text { information } & \text { device } & \text { information }\end{array}$

PMU MU $\quad \begin{gathered}\text { Fault } \\ \text { Recorder }\end{gathered}$

$\begin{array}{cccc}\begin{array}{c}\text { Redundant } \\ \text { information }\end{array} & & & \\ & \begin{array}{c}\text { Energy } \\ \text { metering }\end{array} & \begin{array}{c}\text { Component } \\ \text { protection }\end{array} \\ & & \begin{array}{c}\text { Redundant } \\ \text { information }\end{array} & \end{array}$

FIGURE I. REDUNDANT RELATIONSHIP AMONG MULTIPLE SIGNAL SOURCES IN SMART SUBSTATIONS

\section{Substation Bad Data Identification Method}

The state estimation of the primary system has analyzed the impedance branches such as transformers and transmission lines. The connection lines of the devices in the substation are short, so the substation state estimation analysis no longer considers the impedance of the branches[8].The switch branch of the substation is equivalent to zero-impedance branch, forming a switch island, and the state analysis is performed in the unit of switch island[9].The state estimation of the secondary system is independent of that of the primary system, utilizing linear constraint equations as redundant constraints to restore authentically sampled data[10-12].Based on the state estimation, the bad data identification with the analysis of residual data has a good effect.

\section{A. Smart Substation Information Redundancy Relationship}

In order to ensure that the sampling information error identification can be applied to the protection device, only the redundant relationship between the component protection and the PMU(Phase Measurement Unit) in Figure I can be used to perform state estimation analysis of the substation to identify error information.

The smart substation outdoor installation protection device has the advantages of optimizing the secondary system and improving the reliability of the device[13-15].Its important feature is that it has the ability to interact with SV/GOOSE network of the outdoor installation protection unit and can achieve the sharing of sampling information on the entire station. Figure II is a schematic diagram of an outdoor installation protection device for a smart substation. Taking the outdoor installation protection device for analysis, outdoor installation protection devices include Merging Units, outdoor installation protection units, and Intelligent Terminals, where the MUs (Merging Units) are connected to the transformers and the SV network, and the Intelligent Terminals are connected to the
GOOSE network. The main protection of the protection device avoids the intermediate network transmission process and can effectively improve the rapidity and reliability of protection. At the same time, the Intelligent Terminals and the MUs are respectively connected to the SV and the GOOSE network, in which the Intelligent Terminal can upload the adoption information of the local interval to the SV network, and download the sampling information needed by the protection device to achieve protection functions, and the MU can upload the action information of the local interval and the action commands of the neighboring interval required to the GOOSE network to implement protection functions.

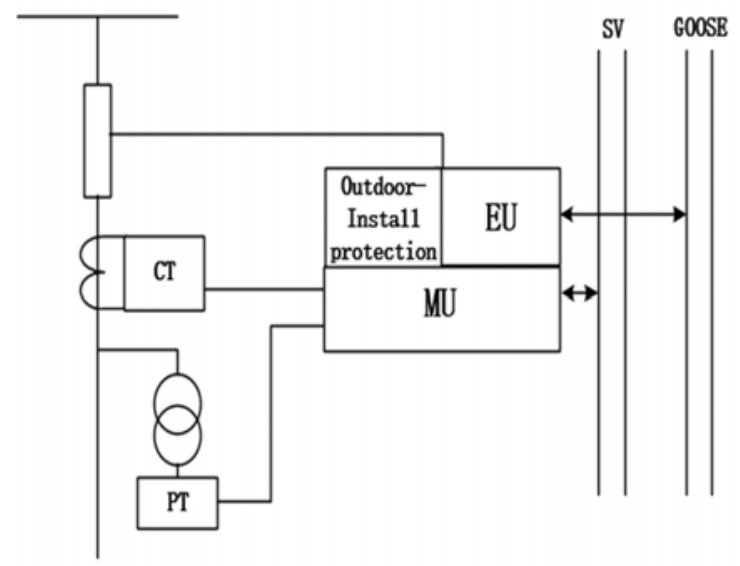

FIGURE II. OUTDOOR INSTALLATION PROTECTION DEVICE FOR SMART SUBSTATION

Through the interaction of the network layer, the information redundancy of the local interval can be successfully implemented. For any position of the substation, different protection devices have their own CT and PT for sampling. When the circuit breaker is closed, two sides of the circuit breaker must also have multiple sampling devices. The information on the multiple sampling devices is identically acquired for the redundancy measurement of the same site, meeting the requirements of information foundation for state estimation method.

\section{B. Phase Voltage Bad Data Identification}

In the secondary information voltage state estimation of the smart substation, considering the zero-impedance model of the substation, the phase voltage in the switch island is taken as the state quantity, and the measurement equations of the voltage amplitude and phase angle are constructed[16]. For PTs (voltage transformers), there is only one coil on the same location, and the dual configuration of the transformer needs to be taken into consideration, and the voltage sampling information on the PMU is used to form a redundancy relationship of the voltage measurement information.

The voltage amplitude state analysis equation built in an electrical island as:

$$
Z_{U^{\varphi}}=1_{m \times 1} U^{\varphi}+v_{U^{\varphi}}
$$


In which: $Z_{U^{\varphi}}$ is the voltage amplitude measurement from different voltage transformers in the electric island, including the voltage measurement of the protection device and the PMU. In order to ensure the redundancy of the measurement information, the voltage transformer can be dually sampled. $1_{m \times 1}$ is a unit column vector whose dimension is $\mathrm{m} \times 1 ; U^{\varphi}$ is the state quantity of the voltage amplitude of the electric island; $v_{U^{\varphi}}$ is the corresponding measurement error.

Similar to the amplitude relationship, the voltage phase angle of the electric island has a similar measurement equation as:

$$
Z_{\theta^{\varphi}}=1_{m \times 1} \theta^{\varphi}+v_{\theta^{\varphi}}
$$

In which: $Z_{\theta^{\varphi}}$ is the voltage phase angle measurement from the protection device and the PMU, which is the same as the amplitude and has a redundancy relationship with it; $\theta^{\varphi}$ is the voltage phase angle state quantity; $v_{\theta^{\varphi}}$ is the corresponding phase angle measurement error.

$i_{d}$ is the No. $i_{d}$ island in the substation and all the island possesses the same measurement equation. The physical constraints are: the phase voltage measurement of the same island measures the same, and the solving formula of the voltage amplitude and phase angle can be obtained based on the least square method.

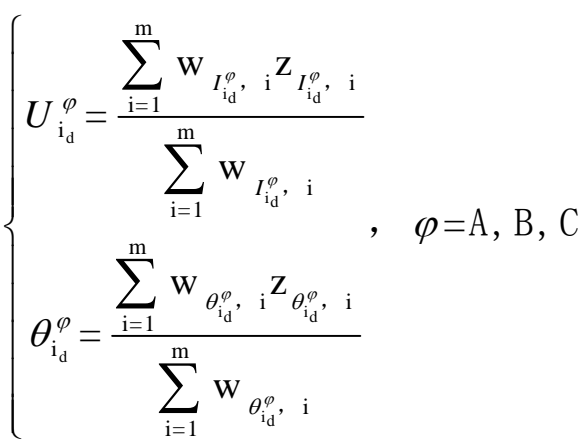

The solving formula for the voltage amplitude and phase angle is essentially a weighted average of the measured values and it is a first-order linear equation. It requires no iteration during the solving process, with fast calculation speed, no convergence problem, and fully meets the protection calculation speed requirements. In the state analysis process, residual equations for each measurement are included. Through calculation and analysis of residuals, it is possible to judge whether there are bad data, and it can be extracted and then recalculated if there is, thereby improving the information accuracy and protection reliability.

\section{Phase Current Bad Data Identification}

The phase current measurement information comes from different coils, constituting a redundant relationship to the current information in the PMU.

According to KCL, there is: $\sum \dot{I}=0$. Similar to the voltage measurement equation analysis method, the phase current of each branch of the node is taken as the state quantity, and the measured value of the phase current is sampled from the protection devices and PMU, and then the measurement equations of the phase current amplitude and phase angle are constructed.

In the electric island, the injected current is equal to the outgoing current, and the current flowing through the same node is equal. Based on the least squares method, the solving formula for voltage amplitude and phase angle can be obtained as:

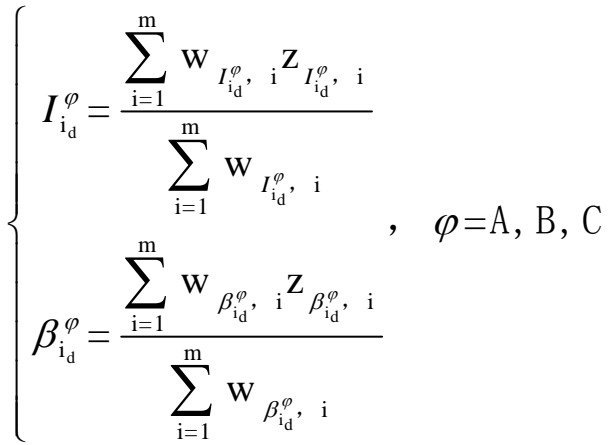

The current measurements of each branch of the same node also constitute a linear relationship. The state estimation of the current is essentially a weighted average of the measured values and it is a first-order linear equation, requires no iteration, has no convergence problem and has fast speed in the solving process, fully meeting the protection calculation requirements. In the state analysis process, residual equations for each measurement are included. Through calculation and analysis of residuals, it is possible to judge whether there are bad data, and it can be extracted and then recalculated if there is, thereby improving the information accuracy and protection reliability.

\section{RELAy PRotection FAULT-TOLERANCE METHOD}

Utilizing state estimation process, residual equations can be calculated. When the residual error is greater than the measurement error, it can be judged that the error data appear and applying a regularized residual search method can confirm the erroneous quantity measurement and remove it, then restarting to perform state estimation to get the correct measurement[17]. It should be noted that when a fault occurs inside the switch island, the physical constraints on the voltage and current redundant sampling information are destroyed, and the state estimation breaks down, resulting that the fault and the sampling error can't be distinguished. In the case of a fault, the voltage and current are subjected to state estimation analysis. For CT and PT at the same location and in the same phase after the fault, they are all judged to be erroneous in information. But CT and PT at the same location have a low probability of 
sampling errors at the same time, so this feature is used to serve as a criterion for the start-up of the fault-tolerance protection.

The residual equations calculation can be used to find error information. Through the principle that faults can cause the voltage and current of the same phase in the same location to change in association with each other can it be judged whether there is a fault, and a fault-tolerant method of relay protection for smart substations based on measurement equation residual is proposed. The specific algorithm flow is as shown in Figure III. The residual equation analysis in the algorithm is a linear calculation, which does not require iteration and the calculation amount is small. It can be calculated in parallel with the busprotection and line-protection algorithm so that the faulttolerance method does not affect the protection operation speed. The basic steps are as follows:

- Initialization. The protection device is configured and initialized, and the sampling information is networked.

- Collect redundant sampling information. Regarding the protection device as a unit, redundant sampling information is collected through the SV network.

- $\quad$ Perform voltage and current state estimation analysis and residual calculation. Open protection when there is no wrong message. When there is an error message and the "error message" is a voltage or current sampled value from the same location, it is considered to be due to a fault and the protection will be also opened. In the rest of the cases, the weighted least squares method is used for error message rejection, and then the state estimation analysis will continue.

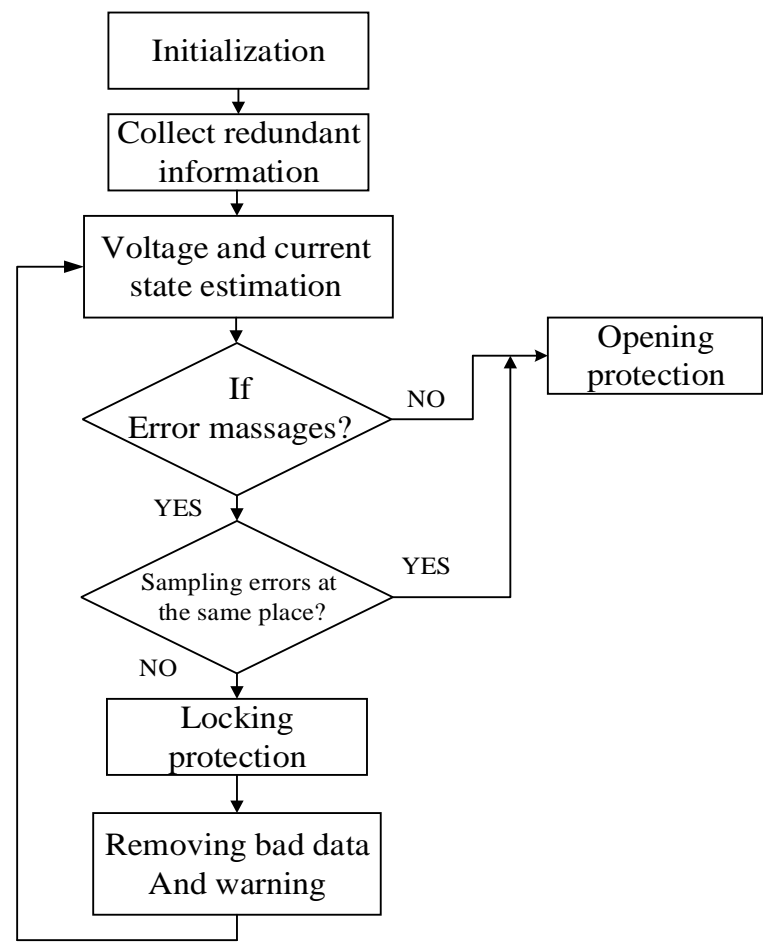

FIGURE III. RELAY PROTECTION FAULT-TOLERANCE ALGORITHM FLOW CHART

\section{ANALYSIS OF EXAMPLES}

Taking the IEEE 10-node system as an example, the simulation analysis is performed to verify the behavior of the line-protection and bus-protection after the fault tolerance method is applied. The simulation system is shown in Figure IV. Among them, Load1 is a smart substation, and SV/GOOSE network is used to share the sampling information of the whole station. It is equipped with PMU, 2 transformers and 6 outlets in the station to analyze the bus-protection and line-protection on Bus Y. Dual configuration of voltage and current transformers are equipped to ensure redundancy measurement of voltage and current.

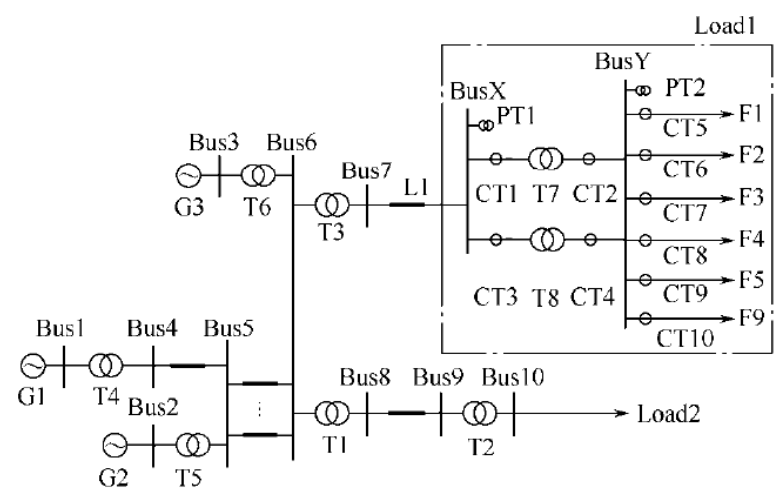

FIGURE IV. TEST SYSTEM STRUCTURE

The probability of sampling errors of multiple trans-formers at the same time is low, and CT and PT errors in the same place at the same time aren't necessary to be taken into account. For the same measurement of the same position, there is only one sampling error being considered. In order to ensure that the error message is sufficient to cause protection refusal, the fault current value is taken as 10 times the normal current and the fault voltage value is taken as zero. The simulation analysis needs to verify whether the error can be identified in the case of sampling information error and ensure that the protection does not malfunction, and that the fault-tolerance method should not affect the protection action in the case of a fault. The simulation analysis of sampling errors in current transformer(CT) on the bus transformer side, current transformer(CT) on the bus outlet side and bus voltage transformer(PT), and of station faults and line faults were performed to verify the effectiveness of the faulttolerance method.

The following five sets of test samples were set in the simulation analysis, and the test results are shown in Table I.

TABLE I. SIMULATION TEST RESULTS

\begin{tabular}{cccc}
\hline No. & Fault? & Errors & Action \\
\hline 1 & NO & CT2 & Not Misoperation \\
2 & NO & CT5 & Not Misoperation \\
3 & NO & PT2 & Not Misoperation \\
4 & BUS ERROR & NO & Correct Action \\
5 & LINE ERROR & NO & Correct Action \\
\hline
\end{tabular}

It can be seen from the results that no matter whether the CT on the transformer side or the line side has a sampling error, the error information can be correctly identified, and the protection can be ensured. When the bus voltage sampling error occurs, the error information can be correctly identified, and the protection 
can be guaranteed. And this method will not affect the normal operation of the protection in the case of a failure.

\section{CONCLUSION}

This article uses the method of system state estimation, utilizing the advantages of information sharing among the intelligent substation network to realize the protection information fault-tolerance method based on measurement equation residual of the secondary system information on the local interval, effectively identifying the error information, avoiding the protection misoperation, and improving the sampling informa-tion accuracy. Through examples, it is verified that this method can effectively avoid the protection misoperation caused by CT sampling errors and transmission distortions, and it can remove bad data, ensure the reliable transmission of information on smart substations and improve the accuracy and credibility of data transmission.

\section{ACKNOWLEDGEMENT}

This work was supported by Scientific and Technical project of State Grid Zhejiang Electric Power Corporation, and the Project No. is: 5211jx150107.

\section{REFERENCE}

[1] Qixun YANG. Microcomputer relay protection foundation[M].Beijing: Water Power Press, 1988.

[2] Deshu CHEN. Computer Relay Protection Principle and Technology[M]. Beijing: Water Power Press, 1992.

[3] Honggen LIAO. Power System Wide Area Protection Communication System[D]. Southwest Jiaotong University, 2008.

[4] Hongen DING, Zemei DAI, Xuesong HUO, et al. Quadratic linear state estimation method based on mixed measurement and its engineering application[J]. Power System Protection and Control, 2016, 44(13): 163170.

[5] Yuan JI, Xin YANG, Yan WANG, et al. Topology identification method based on branch equivalent[J]. Power System Protection and Control, 2015, 43(10): 129-134

[6] Zhichao HUANG, Xia XIE, Bin WANG. Power system state estimation combined with fuzzy comprehensive evaluation and decision-making[J]. Power System Protection and Control, 2015, 43(7): 65-69.

[7] Chunchao HU, Jie HE, Kai MA. Distributed state estimation of power system based on information redundancy[J]. Power System Protection and Control, 2017, 45(16):111-115.

[8] Tao YANG, Hongbin SUN, Anjan B. Two-level PMU-based linear state estimator[C]//Power System Conference and Exposition.Seattle: IEEE/PES, 2009: 1-6.

[9] Hongbin SUN, Boming ZHANG, Niande XIANG. Real-time network analysis switch current and power calculation [J]. Power System Automation, 1996, 20(5): 25-29.

[10] Zhiqiang YAO, Yanping WU, Wei XU, Hao REN, Mingzhi ZHAI, Renhui DOU. Discussion on key technologies for integration of main plant and station for smart grid[J]. Automation of Electric Power Systems, 2017, 41(08): 179-185+191.

[11] Jianglin LI, Daojie ZHANG, Yi ZHAO, Yanpeng ZHANG.Application of distributed data platform in smart substation[J].Power System Protection and Control,2014,42(24):126-131.

[12] Shaoqian HU, Rong HU, Ximing ZHANG, Wenlong WANG, Qing LIN. Development of Intelligent Long-distance Motives[J]. Automation of Electric Power Systems, 2014, 38(09): 119-124.

[13] Fufeng CHEN, Chunlin YU, Hao ZHANG, Yuping LI, Bei DONG, Qi CHEN, Mingjun XUE, Sheng WANG, Xinzhi CHEN, Qian ZHAO. Research on the Localization of Relay Protection in Transformer Substation[J]. Electric Power Automation Equipment, 2017,37 (10): 204210.
[14] Delin WANG, Youtao GENG, Guang LING, Wuluo PAN, Jiayi WU. Comparison of application schemes and economics of plug-and-play localized protection in substations[J]. Automation of Electric Power Systems, 2017, 41(16): 12-19.

[15] Yutao RUAN, Delin WANG, Chen HU, Xintao DONG. Application and Practice of In-place Protection for Unshielded Installation[J]. Power System Protection and Control, 2016, 44(20):1-5.

[16] Qingxin LI, Hongbin SUN, Jing WANG, Boming ZHANG, Wenchuan WU, Qinglai GUO. Three-phase nonlinear state estimation method for substation based on zero impedance branch model[J]. Proceedings of the CSEE, 2011, 31(25): 73-80 .

[17] Boming ZHANG, Shiyu WANG, Niande XIANG. Recursive measurement error estimation identification method and its application [J]. Power System and Automation. 1992, 4(1): 1-8. 